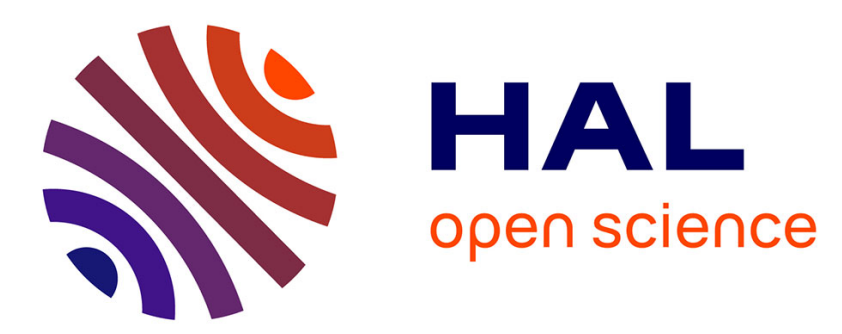

\title{
Finite dimensional approximations for a class of infinite dimensional time optimal control problems
}

\author{
Marius Tucsnak, Julie Valein, Chi-Ting Wu
}

\section{To cite this version:}

Marius Tucsnak, Julie Valein, Chi-Ting Wu. Finite dimensional approximations for a class of infinite dimensional time optimal control problems. International Journal of Control, 2019, 92 (1), pp.132-144. 10.1080/00207179.2016.1228122 . hal-01393258

\section{HAL Id: hal-01393258 \\ https://hal.science/hal-01393258}

Submitted on 7 Nov 2016

HAL is a multi-disciplinary open access archive for the deposit and dissemination of scientific research documents, whether they are published or not. The documents may come from teaching and research institutions in France or abroad, or from public or private research centers.
L'archive ouverte pluridisciplinaire $\mathbf{H A L}$, est destinée au dépôt et à la diffusion de documents scientifiques de niveau recherche, publiés ou non, émanant des établissements d'enseignement et de recherche français ou étrangers, des laboratoires publics ou privés. 


\title{
Finite dimensional approximations for a class of infinite dimensional time optimal control problems
}

\author{
Marius Tucsnak, Julie Valein’ Chi-Ting $\mathrm{Wu}^{\ddagger}$
}

June 24, 2016

\begin{abstract}
In this work we study the numerical approximation of the solutions of a class of abstract parabolic time optimal control problems with unbounded control operator. Our main results assert that, provided that the target is a closed ball centered at the origin and of positive radius, the optimal time and the optimal controls of the approximate time optimal problems converge (in appropriate norms) to the optimal time and to the optimal controls of the original problem. In order to prove our main theorem, we provide a nonsmooth data error estimate for abstract parabolic systems.
\end{abstract}

Keywords. distributed parameter systems, optimal control, numerical approximation AMS subject classifications. 93C25; 93B07; 93C20; 35R30;

\section{Introduction}

Time optimal control of infinite dimensional systems is a subject of growing interest, motivated by numerous applications in domains such as guidance of complex systems or temperature regulation in large buildings. In recent year, using new tools from infinite dimensional systems theory, the literature devoted to this topic grew in a considerable manner (see Arada and Raymond [1], Barbu [4], [5], Fattorini [11], [12], [13], Kunisch and Wang [17], Kunisch and Wachsmuth [16], Li and Yong [21] and Tröltzsch [29]). The specific case of time optimal control for systems governed by parabolic PDE's has numerous applications, from which we quote optimization of building thermal storage (see, for instance [14] and references therein).

The aim of this paper, containing results partially announced in [30], is to study the approximation of the solutions of time optimal (internally or boundary) control problems for a class of infinite dimensional linear systems by projecting the original problem on an appropriate family of finite dimensional spaces. This is a delicate question since, as shown in the above mentioned references, time optimal controls are usually highly oscillating functions (due to the bang-bang property). As far as we know, the only papers having already investigated this issue are Knowles [15], Wang and Wang [32], Wang and Zheng [33] which investigated finite elements approximation for systems governed by the heat

\footnotetext{
*Institut de Mathématiques, Université de Bordeaux, Bordeaux INP, CNRS F-33400 Talence, France, marius.tucsnak@u-bordeaux.fr

${ }^{\dagger}$ Université de Lorraine, Institut Élie Cartan de Lorraine, UMR 7502, Inria, 54506 Vandœuvre-lès-Nancy Cedex, France, julie.valein@univ-lorraine.fr, corresponding author

${ }^{\ddagger}$ Université de Lorraine, Institut Élie Cartan de Lorraine, UMR 7502, Inria, 54506 Vandœuvre-lès-Nancy Cedex, France, chi-ting.wu@univ-lorraine.fr
} 
equation with internal controls. To our knowledge, the similar results obtained in these papers are unknown to boundary time optimal control problems. A similar topic dealing with the homogenization of parabolic system, where we study the asymptotic behavior of solutions and (time-)optimal controls has been developed in recent years (see for example, Carja [8], [9], Castro and Zuazua [10] and Tebou $[27])$.

To state our results we need some notation. Let $X$ and $U$ be real Hilbert spaces, and let $A_{0}$ : $\mathcal{D}\left(A_{0}\right) \rightarrow X$ be a strictly positive operator. It is known that $-A_{0}$ generates an exponentially stable analytic semigroup, denoted by $\mathbb{T}_{t}$. We denote by $\|\|.($ resp. $\langle.,\rangle$.$) the norm (resp. the inner product)$ on $X$. For $\gamma>0$ we denote by $X_{\gamma}$ the space $\mathcal{D}\left(A_{0}^{\gamma}\right)$, endowed with the graph norm. For $\gamma<0$, $X_{\gamma}$ stands for the dual of $X_{-\gamma}$ with respect to the pivot space $X$. We also introduce an operator $B \in \mathcal{L}\left(U, X_{-\alpha}\right)$ with $0 \leqslant \alpha<\frac{1}{2}$, called control operator. In this paper we consider systems of the form

$$
\begin{array}{rlr}
\dot{z}(t)+A_{0} z(t) & =B u(t) \quad(t \geqslant 0), \\
z(0) & =z_{0} \quad\left(z_{0} \in X\right),
\end{array}
$$

where $u \in L^{\infty}([0,+\infty) ; U)$ is the input function and $z$ is the state trajectory. It is well known (this follows, for instance, by combining Theorem 4.4.3 and Proposition 5.1.3 in [31]) that if $z_{0} \in X$ and $u \in L^{\infty}([0,+\infty) ; U)$, there exists a unique solution $z \in C([0,+\infty) ; X)$ of $(1.1)-(1.2)$ and $z$ satisfies

$$
z(t)=\mathbb{T}_{t} z_{0}+\Phi_{t} u, \quad \text { where } \quad \Phi_{t} u=\int_{0}^{t} \mathbb{T}_{t-\sigma} B u(\sigma) \mathrm{d} \sigma
$$

Given $\varepsilon>0$, denote by $\bar{B}(0, \varepsilon)$ the closed ball centered in zero and of radius $\varepsilon$ in $X$. We consider the time optimal control problem which states as follows

(TP) Determine $\tau_{0}^{*}>0$ such that

$$
\tau_{0}^{*}=\min \left\{\tau \geqslant 0 \quad \mid \text { there exists } u \in L^{\infty}([0,+\infty) ; U) \text { s.t. }\|u\|_{L^{\infty}([0,+\infty) ; U)} \leqslant 1 \text { and } z(\tau) \in \bar{B}(0, \varepsilon)\right\},
$$

and the corresponding optimal controls $u_{0}^{*}$.

Denote

$$
\mathcal{U}_{\mathrm{ad}}=\left\{u \in L^{\infty}([0,+\infty) ; U) \mid\|u\|_{L^{\infty}([0,+\infty) ; U)} \leqslant 1\right\} .
$$

It is well-known that the above optimal time $\tau_{0}^{*}$ and optimal control $u_{0}^{*}$ exist under additional assumptions (see, for instance, [23]).

Let $\left(V_{h}\right)_{h>0}$ (resp. $\left.\left(U_{h}\right)_{h>0}\right)$ be a family of finite dimensional subspaces of $X_{\frac{1}{2}}$ (resp. $U$ ), which are normed spaces when endowed with the restriction of the norm of $X_{\frac{1}{2}}$ (resp. $U$ ). We denote $P_{h}$ (resp. $Q_{h}$ ) the orthogonal projector from $X$ onto $V_{h}$ (resp. $U$ onto $U_{h}$ ). For each $h>0$ we consider the following system:

$$
\begin{aligned}
\dot{z}_{h}(t)+A_{h} z_{h}(t) & =B_{h} u_{h}(t) \quad(t \geqslant 0), \\
z_{h}(0) & =P_{h} z_{0},
\end{aligned}
$$

where $u_{h} \in L^{\infty}\left([0,+\infty) ; U_{h}\right),\left(A_{h}\right)_{h>0}$ is defined by

$$
<A_{h} \varphi, \psi>=<A_{0}^{\frac{1}{2}} \varphi, A_{0}^{\frac{1}{2}} \psi>\quad\left(\varphi, \psi \in V_{h}\right),
$$

and $B_{h} \in \mathcal{L}\left(U, V_{h}\right)$ is defined by:

$$
\left\langle B_{h} u, \varphi\right\rangle=\left\langle u, B^{*} \varphi\right\rangle_{U} \quad\left(\varphi \in V_{h}, u \in U\right) .
$$


The above system is the Galerkin approximation of (1.1)-(1.2), and its solution $z_{h}$ can be written as

$$
z_{h}(t)=\mathbb{T}_{t, h} P_{h} z_{0}+\Phi_{t, h} u_{h}, \quad \text { where } \quad \Phi_{t, h} u_{h}=\int_{0}^{t} \mathbb{T}_{t-\sigma, h} B_{h} u_{h}(\sigma) \mathrm{d} \sigma
$$

where $\mathbb{T}_{t, h}=\exp \left(-t A_{h}\right)$ is the semigroup generated by $A_{h}$. Denote by $\bar{B}_{h}(0, \varepsilon)$ the closed ball centered in zero in $V_{h}$ with radius $\varepsilon$. For each $h>0$, we consider the time optimal control problem for the above system (1.5)-(1.6) which states as follows:

$\left(\mathbf{T P}_{\mathbf{h}}\right)$ Determine $\tau_{h}^{*}>0$ such that

$\tau_{h}^{*}=\min \left\{\tau \geqslant 0 \mid\right.$ there exists $u_{h} \in L^{\infty}\left([0,+\infty) ; U_{h}\right)$ s.t. $\left\|u_{h}\right\|_{L^{\infty}\left([0,+\infty) ; U_{h}\right)} \leqslant 1$ and $\left.z_{h}(\tau) \in \bar{B}_{h}(0, \varepsilon)\right\}$, and the corresponding optimal controls $u_{h}^{*}$ in the admissible set $\mathcal{U}_{\mathrm{ad}, h}$, where $\mathcal{U}_{\mathrm{ad}, h}$ is defined by

$$
\mathcal{U}_{\mathrm{ad}, h}=\left\{u \in L^{\infty}\left([0,+\infty) ; U_{h}\right) \mid\|u\|_{L^{\infty}\left([0,+\infty) ; U_{h}\right)} \leqslant 1\right\} .
$$

The goal of this work is to study the convergence of $\tau_{h}^{*}$ to $\tau_{0}^{*}$ and of $u_{h}^{*}$ to $u_{0}^{*}$ when $h \rightarrow 0$. To this aim, we need appropriate assumptions on the approximation properties of the spaces $\left(V_{h}\right)_{h>0}$ and $\left(U_{h}\right)_{h>0}$. More precisely, we assume that there exist $\theta>0, h_{1}>0, C>0$ such that for every $h \in\left(0, h_{1}\right)$ and $0 \leqslant \gamma \leqslant 1$ we have:

$$
\left\|x-P_{h} x\right\| \leqslant C h^{\theta \gamma}\|x\|_{\gamma} \text { for every } x \in X_{\gamma},
$$

$A_{h}$ is uniformly (with respect to $h$ ) analytic, in the sense of the Definition 3.2 below,

$$
\begin{aligned}
& \left\|\left(I-P_{h}\right) B\right\|_{\mathcal{L}(U, X)} \leqslant C h^{\theta(1-\alpha)}, \\
& \left\|P_{h} B\right\|_{\mathcal{L}\left(U, V_{h}\right)} \leqslant C h^{-\theta \alpha}, \\
& \lim _{h \rightarrow 0}\left\|Q_{h} u-u\right\|_{U}=0 \text { for every } u \in U,
\end{aligned}
$$

where $\alpha$ represents the unboundedness degree of the operator $B$ (introduced in the beginning of this section).

We are now in a position to state the main results of this paper:

Theorem 1.1. With the notation and assumptions on operators $A_{0}, B, A_{h}, B_{h}$, assume that $(C 1)$ (C5) hold and that $z_{0} \in X,\left\|z_{0}\right\|>\varepsilon$. Then $\lim _{h \rightarrow 0} \tau_{h}^{*}=\tau_{0}^{*}$.

Theorem 1.2. With the notation and assumptions on operators $A_{0}, B, A_{h}, B_{h}$, assume that $(C 1)$ (C5) hold and that the only $\eta \in X$ for which there exists an open non-empty interval $I$ with $B^{*} \mathbb{T}_{t}^{*} \eta=0$ for $t \in I$ is $\eta=0$. Then we have

$$
u_{h}^{*} \rightarrow u_{0}^{*} \text { strongly in } L^{2}([0, T] ; U),
$$

where $T=2 \frac{\ln \left(\left\|z_{0}\right\| / \varepsilon\right)}{\lambda_{1}}\left(\lambda_{1}\right.$ is the first eigenvalue of the operator $\left.A_{0}\right)$ and extending $\left(u_{h}^{*}\right)_{h}$ and $u_{0}^{*}$ to time $T$ by zero.

It is worth mentioning that similar convergence results have been obtained in [15] and [33]. In [15], the control space takes only in finite dimensional subspaces of $U$ and in [33] the initial data $z_{0}$ is taken in $X_{\frac{1}{2}}$ and with bounded operator $B$, which can not apply to boundary control problem. Our results 
can be seen as generalizations of those obtained in [33], in the sense that we consider a class of abstract problems including the PDE systems studied there. The novelty of our results is that we weaken the regularity assumptions on the initial data with $z_{0} \in X$ and also that we weaken the assumption on control operator $B$ with $B \in \mathcal{L}\left(U, X_{-\alpha}\right), 0 \leqslant \alpha<\frac{1}{2}$.

The outline of the remaining part of this paper is as follows. Section 2 contains some necessary background on time optimal control problems. In Section 3, we provide some error estimate results with smooth initial data which play an essential role to prove our Theorem 1.1. In Section 4, we provide the proof of our main theorems. In Section 5, we apply our abstract results to some equations. Throughout the paper, we denote by $C$ a positive constant that may change from line to line.

\section{Some background on time optimal control problem}

This section, in which we continue to use the notation and assumptions introduced in Section 1 for $X, U, A_{0}, B$, is devoted to some background on the time optimal control problem (TP). We first introduce some basic notions on controllability (see for example Ch6. and Ch11. in [31]). by

For $\tau>0$, we denote $\Psi_{\tau} \in \mathcal{L}\left(X, L^{2}([0, \tau] ; U)\right)$ the observation operator of $\left(A_{0}^{*}, B^{*}\right)$ on $[0, \tau]$ defined

$$
\left(\Psi_{\tau} z_{0}\right)(\sigma)=B^{*} \mathbb{T}_{\sigma}^{*} z_{0} \quad\left(z_{0} \in X, \sigma \in[0, \tau]\right) .
$$

It is clear that (see, for instance, [31, Ch. 4]),

$$
\Phi_{\tau}^{*}=R_{\tau} \Psi_{\tau} \quad(\tau>0),
$$

where $R_{\tau}$ is the reflection operator on $L^{2}([0, \tau] ; U)$ defined by $R_{\tau} u(t)=u(\tau-t)$.

We then recall the following existence result, which can be proved by using standard techniques providing the existence of time optimal controls for linear systems. We refer to [12] or Lions [22] for a detailed description of the methodology or to Micu et al [23, Proposition 2.6] for a slightly more general version of this result.

Proposition 2.1. For every $z_{0} \in X$ and $\varepsilon>0$, the time optimal control problem (TP) admits at least one solution $\left(\tau^{*}, u^{*}\right)$.

We need below a special version of the maximum principle. Although maximum principle is a classical tool in optimal control problems, we were unable to find the version we need (abstract setting with unbounded control operators) in the existing literature. Therefore we give the precise statement and a short proof below.

Theorem 2.2. With the notation of Proposition 2.1, assume that $\tau^{*}>0$. Then the time optimal control $u^{*}$ satisfies the maximum principle, i.e., there exists $\xi \in X, \xi \neq 0$ such that,

$$
\left\langle u^{*}(\sigma),\left(\Psi_{\tau^{*}} \xi\right)\left(\tau^{*}-\sigma\right)\right\rangle_{U}=\max _{v \in U,\|v\| \leqslant 1}\left\langle v,\left(\Psi_{\tau^{*}} \xi\right)\left(\tau^{*}-\sigma\right)\right\rangle_{U}, \quad\left(\sigma \in\left(0, \tau^{*}\right) \text { a.e. }\right) .
$$

Moreover, $\xi$ satisfies the transversality condition, i.e.:

$$
\left\langle\xi, z-\mathbb{T}_{\tau^{*}} z_{0}-\Phi_{\tau^{*}} u^{*}\right\rangle \geqslant 0 \quad(z \in \bar{B}(0, \varepsilon)) .
$$

Proof. Let $\left(\tau^{*}, u^{*}\right)$ be a solution of the time optimal control in Proposition 2.1 and let

$$
z_{1}=\mathbb{T}_{\tau} * z_{0}+\Phi_{\tau} * u^{*}
$$


Moreover, for each $z_{0} \in X$ and $\tau>0$ denote

$$
U_{\tau}:=\left\{\left.u\right|_{[0, \tau]} \mid u \in \mathcal{U}_{\mathrm{ad}}\right\} \text { and } \mathcal{R}_{\tau} z_{0}=\mathbb{T}_{\tau} z_{0}+\Phi_{\tau} U_{\tau}
$$

We claim that

$$
z_{1} \in \partial \mathcal{R}_{\tau} * z_{0} \cap \partial B(0, \varepsilon)
$$

Indeed, it can be easily checked that we necessarily have $\left\|z_{1}\right\|=\varepsilon$. If we admit, by contradiction, that $z_{1}$ is an interior point of $\mathcal{R}_{\tau} * z_{0}$, this implies that there exists $z_{2} \in \mathcal{R}_{\tau} * z_{0}$ with $\left\|z_{2}\right\|<\varepsilon$. Consequently, there exists $u_{2} \in U_{\tau^{*}}$ such that

$$
\mathbb{T}_{\tau^{*} z_{0}}+\Phi_{\tau^{*} u_{2}}=z_{2}
$$

Since the map $t \mapsto\left\|\mathbb{T}_{t} z_{0}+\Phi_{t} u_{2}\right\|$ is continuous and that $\tau^{*}>0$, we obtain that there exists $\tau \in\left(0, \tau^{*}\right)$ such that

$$
\left\|\mathbb{T}_{\tau} z_{0}+\Phi_{\tau} u_{2}\right\|=\varepsilon
$$

This contradicts the fact that $\left(\tau^{*}, u^{*}\right)$ is a solution of our time optimal control problem. We have thus proved our claim (2.14).

Thus we clearly have that the sets $\mathcal{R}_{\tau} * z_{0}$ and $B(0, \varepsilon)$ are non-empty, convex and they have no common point. Moreover, since $B(0, \varepsilon)$ is open, we can apply the geometric version of the Hahn Banach theorem (see, for instance [7, Theorem 1.6]) to obtain that there exists a hyperplane separating $\mathcal{R}_{\tau} * z_{0}$ and $B(0, \varepsilon)$. This means that there exists $\alpha \in \mathbb{R}$ and $\xi \in X, \xi \neq 0$ such that

$$
\begin{array}{ll}
\langle\xi, \eta\rangle \leqslant \alpha & \left(\eta \in \mathcal{R}_{\tau^{*} z_{0}}\right), \\
\langle\xi, \eta\rangle \geqslant \alpha \quad(\eta \in B(0, \varepsilon)) .
\end{array}
$$

The two above inequalities and (2.14) imply that

$$
\left\langle\xi, z_{1}\right\rangle=\alpha
$$

From (2.15) and (2.17) we deduce that

$$
\left\langle\xi, z_{1}\right\rangle \geqslant\langle\xi, \eta\rangle \quad\left(\eta \in \mathcal{R}_{\tau^{*}} z_{0}\right)
$$

which combined with the duality (2.11), clearly implies the maximum principle (2.12).

Moreover, we easily deduce the transversality condition (2.13) from (2.16) and (2.17).

Remark 2.3. To be more precise, we can deduce from the transversality condition (2.13) that:

$$
\xi=-k\left(\mathbb{T}_{\tau^{*}} z_{0}+\Phi_{\tau^{*}} u^{*}\right), \quad \text { for some positive constant } k .
$$

Corollary 2.4. With the notation of Theorem 2.2, assume that $\tau^{*}>0$ and that the assumption of Theorem 1.2 holds, i.e., the only $\eta \in X$ for which there exists an open non-empty interval I with $B^{*} \mathbb{T}_{t}^{*} \eta=0$ for $t \in I$ is $\eta=0$. Then the time optimal control $u^{*}$ is unique and it has the bang-bang property:

$$
\left\|u^{*}(t)\right\|=1 \quad\left(t \in\left(0, \tau^{*}\right) \text { a.e. }\right) .
$$


Proof. We first remark that the following statement holds: If $\eta \in X$ such that there exists a subset $e \subset[0, \tau]$ of positive measure with $B^{*} \mathbb{T}_{t}^{*} \eta=0$ for $t \in e$, then $\eta=0$. Indeed, the facts that the map $t \mapsto B^{*} \mathbb{T}_{t}^{*} \eta$ is analytic (which is due to the analyticity of $\mathbb{T}_{t}$ ) and that it vanishes in $e$ imply that this map vanishes for $t$ in some non-empty open interval $I$. Therefore, according to the hypothesis, we have $\eta=0$.

With the above property, it is clear that $\left(\Psi_{\tau^{*}} \xi\right)\left(\tau^{*}-t\right) \neq 0$ for almost every $t \in\left(0, \tau^{*}\right)$. Indeed, if it is not the case, we deduce from the above property that $\xi=0$, which contradicts Theorem 2.2.

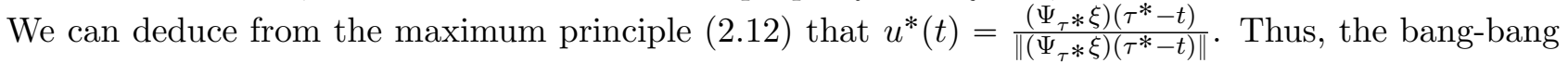
property of the time-optimal control holds.

The uniqueness of the time-optimal control can be deduced from the strict convexity of $U$. This ends the proof.

\section{Nonsmooth data error estimates for abstract parabolic equations}

In this section, we gather, for easy reference, some results providing error estimates for the semidiscrete Galerkin approximation of some abstract parabolic systems. Our results are strongly inspired by Lasiecka and Triggiani [20, Ch.4] and Badra [3, Ch.5], where they deal with a more general framework. We continue to use the same notation and assumption introduced in Section 1 for $X, U, A_{0}, B, A_{h}$, $B_{h}$ and $V_{h}$ and we recall that $\mathbb{T}_{t}$ (resp. $\mathbb{T}_{t, h}$ ) is the semigroup generated by $-A_{0}$ (resp. $-A_{h}$ ). We then consider the following linear equation and its approximated scheme (where we do not approximate $u$ by $\left.u_{h}\right)$ :

$$
\begin{aligned}
\dot{z}(t)+A_{0} z(t) & =B u(t) \quad(t \geqslant 0), \\
z(0) & =z_{0} \in X, \\
\dot{z}_{h}(t)+A_{h} z_{h}(t) & =B_{h} u(t) \quad(t \geqslant 0), \\
z_{h}(0) & =P_{h} z_{0},
\end{aligned}
$$

with $u \in L^{\infty}([0,+\infty) ; U)$. We first recall a classical characterization of analytic semigroups:

Proposition 3.1. $\mathbb{T}_{t}$ is an analytic semigroup if and only if there exist $C, \delta>0$ such that

$$
\Sigma \subset \rho\left(A_{0}\right) \text { and }\left\|\left(\lambda I-A_{0}\right)^{-1}\right\|_{\mathcal{L}(X)} \leqslant \frac{C}{|\lambda|} \quad(\lambda \in \Sigma),
$$

where $\Sigma$ is defined by $\Sigma:=\left\{\lambda \in \mathbb{C}|| \arg (\lambda) \mid \leqslant \frac{\pi}{2}+\delta\right\}$.

It is well known (see, for instance, $\left[24\right.$, Ch. 2.6]) that if $\mathbb{T}_{t}$ is analytic then

$$
\left\|A_{0}^{\gamma} \mathbb{T}_{t}\right\|_{\mathcal{L}(X)} \leqslant \frac{C}{t^{\gamma}} \quad(t \geqslant 0, \gamma \in(0,1))
$$

We also need the concept of uniform analyticity of the family of semigroups $\left(\mathbb{T}_{t, h}\right)_{h \geqslant 0}$.

Definition 3.2. $\mathbb{T}_{t, h}$ is an uniformly analytic semigroup if there exist $C$ independant of $h, \delta>0$ such that for every $h>0$ we have

$$
\Sigma \subset \rho\left(A_{h}\right) \text { and }\left\|\left(\lambda I-A_{h}\right)^{-1}\right\|_{\mathcal{L}(X)} \leqslant \frac{C}{|\lambda|} \quad(\lambda \in \Sigma),
$$

where $\Sigma$ is defined in Proposition 3.1. 
Similarly, the above definition implies the following property:

$$
\left\|A_{h}^{\gamma} \mathbb{T}_{t, h}\right\|_{\mathcal{L}(X)} \leqslant \frac{C}{t^{\gamma}} \quad(t>0, \gamma \in[0,1]) .
$$

It is also clear that

$$
B_{h} u=\left(P_{h} B\right) u \quad(u \in U),
$$

and (see assumption $(C 4)$ )

$$
\left\|B_{h}\right\|_{\mathcal{L}\left(U, V_{h}\right)} \leqslant C h^{-\theta \alpha} .
$$

We recall in the following some well-known results:

Lemma 3.3. Assume that $\mathbb{T}_{t}$ is analytic. Then, for any $0 \leqslant \gamma \leqslant 1$, there exists $C>0$ such that the following estimates hold:

1. $\left\|A_{0}^{\gamma}\left(\lambda I-A_{0}\right)^{-1}\right\|_{\mathcal{L}(X)} \leqslant \frac{C}{|\lambda|^{1-\gamma}} \quad(\lambda \in \Sigma)$.

2. $\mathbb{T}_{t} z=\frac{1}{2 \pi i} \int_{\Gamma} e^{\lambda t}\left(\lambda I-A_{0}\right)^{-1} z \mathrm{~d} \lambda \quad(z \in X)$, where $\Gamma$ is the path composed from the two rays $\rho e^{i \psi}$ and $\rho e^{-i \psi}, 0<\rho<\infty$ and $\pi / 2<\psi<\pi / 2+\delta$, $\Gamma$ being oriented so that $\Im \lambda$ increases along $\Gamma$ ( $\Im \lambda$ denotes the imaginary part of $\lambda$ ).

Proof. The proof of the first estimate can be found in [24, Ch. 2.6] and the proof of the last assertion can be found in $[24$, Th. 7.7, Ch. 1].

Similar to Lemma 3.3, it is clear that we have the following lemma:

Lemma 3.4. Assume that $\mathbb{T}_{t, h}$ is uniformly analytic. Then, for any $0 \leqslant \gamma \leqslant 1$, there exists $C>0$ such that the following statement hold:
1. $\left\|A_{h}^{\gamma}\left(\lambda I-A_{h}\right)^{-1}\right\|_{\mathcal{L}(X)} \leqslant \frac{C}{|\lambda|^{1-\gamma}} \quad(\lambda \in \Sigma)$.
2. $\mathbb{T}_{t, h} z=\frac{1}{2 \pi i} \int_{\Gamma} e^{\lambda t}\left(\lambda I-A_{h}\right)^{-1} z \mathrm{~d} \lambda \quad\left(z \in V_{h}\right)$.

We gather in the proposition below, with no claim of originality, several error estimates which play a central role in the proof of our main result. Some of the proofs are very similar to those in [3, Ch.5], so that they are detailed.

Proposition 3.5. Assume that the assumption $(C 1)-(C 4)$ hold. Then, there exists $C>0$, such that for every $h>0$, we have the following properties:

1. $\left\|A_{0}^{-1}-A_{h}^{-1} P_{h}\right\|_{\mathcal{L}(X)} \leqslant C h^{\theta}$.

2. $\left\|A_{0}^{-1}\left(B-B_{h}\right)\right\|_{\mathcal{L}(U, X)} \leqslant C h^{\theta(1-\alpha)}$.

3. $\left\|\left(\lambda I-A_{0}\right)^{-1}-\left(\lambda I-A_{h}\right)^{-1} P_{h}\right\|_{\mathcal{L}(X)} \leqslant C h^{\theta} \quad(\lambda \in \Sigma)$.

4. $\left\|\left(\lambda I-A_{0}\right)^{-1} B-\left(\lambda I-A_{h}\right)^{-1} B_{h}\right\|_{\mathcal{L}(U, X)} \leqslant C h^{\theta(1-\alpha)} \quad(\lambda \in \Sigma)$.

5. $\left\|\mathbb{T}_{t, h} P_{h}-\mathbb{T}_{t}\right\|_{\mathcal{L}(X)} \leqslant C t^{-1} h^{\theta}$.

6. $\left\|\mathbb{T}_{t, h} B_{h}-\mathbb{T}_{t} B\right\|_{\mathcal{L}(U, X)} \leqslant C t^{-1} h^{\theta(1-\alpha)}$.

7. $\left\|\mathbb{T}_{., h} B_{h}-\mathbb{T} . B\right\|_{L^{1}([0, t] ; \mathcal{L}(U, X))} \leqslant C h^{\theta(1-\alpha)}(|\ln h|+t+1)$.

\section{Proof.}

1. We skip this proof which is based on Proposition 5.1.1 and Proposition 5.1.3 in [20].

2. We skip this proof as it is similar to the proof of previous assertion by using assumption $(C 3)$. 
3. Notice at first that by simple calculation, it is clear that we have

$$
\left(\lambda I-A_{0}\right)^{-1}=-A_{0}^{-1}+\lambda\left(\lambda I-A_{0}\right)^{-1} A_{0}^{-1}
$$

and

$$
\left(\lambda I-A_{h}\right)^{-1} P_{h}=-A_{h}^{-1} P_{h}+\lambda\left(\lambda I-A_{h}\right)^{-1} A_{h}^{-1} P_{h} .
$$

Denote $M_{h}(\lambda)=\left(\lambda I-A_{0}\right)^{-1}-\left(\lambda I-A_{h}\right)^{-1} P_{h}$, we then have:

$$
\begin{aligned}
M_{h}(\lambda)= & -A_{0}^{-1}+A_{h}^{-1} P_{h}+\lambda\left(\lambda I-A_{0}\right)^{-1} A_{0}^{-1}-\lambda\left(\lambda I-A_{h}\right)^{-1} A_{h}^{-1} P_{h} \\
= & -\left(A_{0}^{-1}-A_{h}^{-1} P_{h}\right)+\lambda\left(\lambda I-A_{0}\right)^{-1}\left(A_{0}^{-1}-A_{h}^{-1} P_{h}\right)+\lambda\left(\lambda I-A_{0}\right)^{-1} A_{h}^{-1} P_{h} \\
& -\lambda\left(\lambda I-A_{h}\right)^{-1} A_{h}^{-1} P_{h} \\
= & -\left(I-\lambda\left(\lambda I-A_{0}\right)^{-1}\right)\left(A_{0}^{-1}-A_{h}^{-1} P_{h}\right)+\lambda M_{h}(\lambda) A_{h}^{-1} P_{h} .
\end{aligned}
$$

This leads to $M_{h}(\lambda)=-\left(I-\lambda\left(\lambda I-A_{0}\right)^{-1}\right)\left(A_{0}^{-1}-A_{h}^{-1} P_{h}\right)\left(I-\lambda A_{h}^{-1} P_{h}\right)^{-1}$.

Notice that

$$
\left(I-\lambda A_{h}^{-1} P_{h}\right)^{-1}=I-P_{h}-A_{h}\left(\lambda I-A_{h}\right)^{-1} P_{h} .
$$

Indeed, we have

$$
\begin{aligned}
& \left(I-\lambda A_{h}^{-1} P_{h}\right)\left(I-P_{h}-A_{h}\left(\lambda I-A_{h}\right)^{-1} P_{h}\right) \\
& =I-P_{h}-A_{h}\left(\lambda I-A_{h}\right)^{-1} P_{h}-\lambda A_{h}^{-1} P_{h}+\lambda A_{h}^{-1} P_{h}+\lambda A_{h}^{-1} A_{h}\left(\lambda I-A_{h}\right)^{-1} P_{h} \\
& =I-P_{h}+\left(\lambda I-A_{h}\right)\left(\lambda I-A_{h}\right)^{-1} P_{h}=I .
\end{aligned}
$$

In the same manner, $\left(I-P_{h}-A_{h}\left(\lambda I-A_{h}\right)^{-1} P_{h}\right)\left(I-\lambda A_{h}^{-1} P_{h}\right)=I$. Thus, (3.8) holds.

By analyticity assumption (3.5) and assertion 1. in Lemma 3.4, we know that there exists a constant $C$ independent of $\lambda$ and $h$ such that,

$$
\left\|\lambda\left(\lambda I-A_{0}\right)^{-1}\right\|_{\mathcal{L}(X)} \leqslant C \frac{|\lambda|}{|\lambda|}=C \quad \text { and } \quad\left\|A_{h}\left(\lambda I-A_{h}\right)^{-1}\right\| \leqslant C \quad(\lambda \in \Sigma),
$$

which leads to, using assertion 1. of Proposition 3.5

$$
\left\|M_{h}(\lambda)\right\|_{\mathcal{L}(X)} \leqslant\left\|I-\lambda\left(\lambda I-A_{0}\right)^{-1}\right\|_{\mathcal{L}(X)} C h^{\theta}\left\|I-P_{h}-A_{h}\left(\lambda I-A_{h}\right)^{-1} P_{h}\right\|_{\mathcal{L}(X)} \leqslant C h^{\theta} .
$$

This ends the proof of assertion 3..

4. Denote $M_{h, B}(\lambda)=\left(\lambda I-A_{0}\right)^{-1} B-\left(\lambda I-A_{h}\right)^{-1} B_{h}$. Notice that $\left(\lambda I-A_{0}\right)^{-1}=A_{0}\left(\lambda I-A_{0}\right)^{-1} A_{0}^{-1}$. Then, we have:

$$
\begin{aligned}
M_{h, B}(\lambda) & =\left(\lambda I-A_{0}\right)^{-1}\left(B-B_{h}\right)+\left(\left(\lambda I-A_{0}\right)^{-1}-\left(\lambda I-A_{h}\right)^{-1}\right) B_{h} \\
& =A_{0}\left(\lambda I-A_{0}\right)^{-1} A_{0}^{-1}\left(B-B_{h}\right)+\left(\left(\lambda I-A_{0}\right)^{-1}-\left(\lambda I-A_{h}\right)^{-1}\right) B_{h} .
\end{aligned}
$$

Since $A_{0}\left(\lambda I-A_{0}\right)^{-1}$ is a bounded operator (by assertion 1 . in Lemma 3.3), it is clear that by using assertions 2. and 3. of Proposition 3.5 and assumption $(C 4)$, we have:

$$
\begin{aligned}
\left\|M_{h, B}(\lambda)\right\|_{\mathcal{L}(U, X)} & \leqslant C\left\|A_{0}^{-1}\left(B-B_{h}\right)\right\|_{\mathcal{L}(U, X)}+\left\|\left(\lambda I-A_{0}\right)^{-1}-\left(\lambda I-A_{h}\right)^{-1}\right\|_{\mathcal{L}(X)}\left\|B_{h}\right\|_{\mathcal{L}\left(U, V_{h}\right)} \\
& \leqslant C h^{\theta(1-\alpha)}+C h^{\theta}\left\|B_{h}\right\|_{\mathcal{L}\left(U, V_{h}\right)} \leqslant C h^{\theta(1-\alpha)} .
\end{aligned}
$$


5. It is clear that, by using assertion 2. in Lemma 3.3 and assertion 2. in Lemma 3.4, we have:

$$
\mathbb{T}_{t}-\mathbb{T}_{t, h} P_{h}=\frac{1}{2 \pi i} \int_{\Gamma} e^{\lambda t}\left(\left(\lambda I-A_{0}\right)^{-1}-\left(\lambda I-A_{h}\right)^{-1} P_{h}\right) \mathrm{d} \lambda .
$$

Then, by using assertion 3. in Proposition 3.5, we have:

$$
\left\|\mathbb{T}_{t}-\mathbb{T}_{t, h} P_{h}\right\|_{\mathcal{L}(X)}=C\left(\int_{\Gamma}\left|e^{\lambda t}\right| \mathrm{d} \lambda\right) h^{\theta} \leqslant C t^{-1} h^{\theta}
$$

This ends the proof.

6. We skip this proof as it is similar to the proof of the previous assertion by using assertion 4. in Proposition 3.5.

7. We calculate:

$$
\left\|\mathbb{T}_{., h} B_{h}-\mathbb{T} . B\right\|_{L^{1}([0, t] ; \mathcal{L}(U, X))}=\int_{0}^{h^{\theta}}\left\|\mathbb{T}_{\sigma, h} B_{h}-\mathbb{T}_{\sigma} B\right\|_{\mathcal{L}(U, X)} \mathrm{d} \sigma+\int_{h^{\theta}}^{t}\left\|\mathbb{T}_{\sigma, h} B_{h}-\mathbb{T}_{\sigma} B\right\|_{\mathcal{L}(U, X)} \mathrm{d} \sigma=I_{1}+I_{2}
$$

By using assertion 6. of Proposition 3.5, we have:

$$
I_{2} \leqslant C h^{\theta(1-\alpha)}\left(\int_{h^{\theta}}^{t} \sigma^{-1} \mathrm{~d} \sigma\right) \leqslant C h^{\theta(1-\alpha)}\left(\int_{h^{\theta}}^{1} \sigma^{-1} \mathrm{~d} \sigma+\int_{1}^{t} 1 \mathrm{~d} \sigma\right) \leqslant C h^{\theta(1-\alpha)}(|\ln h|+t+1) .
$$

Note that the assumption $B \in \mathcal{L}\left(U, X_{-\alpha}\right)$ means that $A_{0}^{-\alpha} B$ is a bounded operator in $\mathcal{L}(U, X)$. Then, in order to deal with $I_{1}$, using also the analyticity of $\mathbb{T}$ (see (3.5)), for $h$ small enough, we have, for every $0 \leqslant \alpha<\frac{1}{2}$,

$$
\left\|\mathbb{T}_{\sigma} B\right\|_{\mathcal{L}(U, X)} \leqslant\left\|\mathbb{T}_{\sigma} A_{0}^{\alpha}\right\|_{\mathcal{L}(X)}\left\|A_{0}^{-\alpha} B\right\|_{\mathcal{L}(U, X)} \leqslant C \sigma^{-\alpha}
$$

Similarly, by using again the assumption $B \in \mathcal{L}\left(U, X_{-\alpha}\right)$, it is clear that $A_{h}^{-\alpha} B_{h}$ is a bounded operator in $\mathcal{L}(U, X)$. Thus, we have

$$
\left\|\mathbb{T}_{\sigma, h} B_{h}\right\|_{\mathcal{L}(U, X)} \leqslant\left\|\mathbb{T}_{\sigma, h} A_{h}^{\alpha}\right\|_{\mathcal{L}(U, X)}\left\|A_{h}^{-\alpha} B_{h}\right\|_{\mathcal{L}(U, X)} \leqslant C \sigma^{-\alpha},
$$

using (3.7).

Finally, by the two previous inequalities, we have :

$$
I_{1} \leqslant C \int_{0}^{h^{\theta}} \sigma^{-\alpha} \mathrm{d} \sigma \leqslant C h^{\theta(1-\alpha)}
$$

Thus, we have

$$
\left\|\mathbb{T}_{., h} B_{h}-\mathbb{T} \cdot B\right\|_{L^{1}([0, t] ; \mathcal{L}(U, X))} \leqslant C h^{\theta(1-\alpha)}(|\ln h|+t+1) .
$$

This ends the proof.

We will now present the main result of this section which gives us a nonsmooth initial data error estimate. 
Theorem 3.6. Let $z_{0} \in X$, let $z$ be the solution of (3.1),(3.2) and let $z_{h}$ be the solution of (3.3), (3.4). Moreover, assume that $(C 1)-(C 4)$ hold. Then, there exist $C>0, \tilde{h}>0$ such that for $h \in(0, \tilde{h})$ we have the following error estimate:

$$
\left\|z(t)-z_{h}(t)\right\|_{X} \leqslant C h^{\theta} t^{-1}\left\|z_{0}\right\|_{X}+C h^{\theta(1-\alpha)}(|\ln h|+t+1)\|u\|_{L^{\infty}([0,+\infty) ; U)}, \quad \forall t>0 .
$$

Proof. Denote $K_{h}(t)=\mathbb{T}_{t} B-\mathbb{T}_{t, h} B_{h}$.

We recall that $z$, solution of system (1.1)-(1.2), satisfies (1.3) and $z_{h}$, solution of (1.5)-(1.6), satisfies (1.9). It is clear that

$$
\left\|z(t)-z_{h}(t)\right\|_{X} \leqslant\left\|\mathbb{T}_{t} z_{0}-\mathbb{T}_{t, h} P_{h} z_{0}\right\|_{X}+\left\|\int_{0}^{t} K_{h}(t-\sigma) u(\sigma) \mathrm{d} \sigma\right\|_{X} .
$$

By using assertions 5. and \%. in Proposition 3.5, we have:

$$
\begin{aligned}
\left\|z(t)-z_{h}(t)\right\|_{X} & \leqslant C h^{\theta} t^{-1}\left\|z_{0}\right\|_{X}+\left\|K_{h} * u\right\|_{L^{1}([0, t] ; X)} \\
& \leqslant C h^{\theta} t^{-1}\left\|z_{0}\right\|_{X}+\left\|K_{h}\right\|_{L^{1}([0, t] ; \mathcal{L}(U, X))}\|u\|_{L^{\infty}([0, t] ; U)} \\
& \leqslant C h^{\theta} t^{-1}\left\|z_{0}\right\|_{X}+C h^{\theta(1-\alpha)}(|\ln h|+t+1)\|u\|_{L^{\infty}([0, t] ; U)} .
\end{aligned}
$$

This ends the proof.

\section{Proof of the main results}

We denote $\tau_{0}^{*}\left(z_{0}\right)$ (resp. $\tau_{h}^{*}\left(z_{0, h}\right)$ ) the optimal time associated with the initial state $z_{0} \in X$ (resp. $z_{0, h} \in V_{h}$ ) and with final state in $\bar{B}(0, \epsilon)$ (resp. $\overline{B_{h}}(0, \varepsilon)$ ). We use also the notation $z\left(t, z_{0}, u\right)$ for the solution of the system (1.1)-(1.2) (resp. $z_{h}\left(t, z_{0, h}, u_{h}\right)$ for the system (1.5)-(1.6)) associated with the initial state $z_{0}$ (resp. $z_{0, h}$ ) and with the control $u$ (resp. $u_{h}$ ) at time $t$.

We first recall a generalized Aubin-Lions theorem (Theorem 4.1) and a standard energy estimate (Lemma 4.2).

Theorem 4.1. Let $T>0$ and let $Y_{0}, Y_{1}, Y_{2}$ be Banach spaces such that $Y_{0} \subset Y_{1} \subset Y_{2}$. Assume that $Y_{i}$ is reflexive for $i=0,1,2$ and that the embedding of $Y_{0}$ into $Y_{1}$ is compact. Let $1<r<\infty$.

Then, the space

$$
W=L^{\infty}\left([0, T] ; Y_{0}\right) \cap W^{1, r}\left([0, T] ; Y_{2}\right)
$$

is compactly embedded in $C\left([0, T] ; Y_{1}\right)$.

Proof. See for example [26, Cor. 4, p.85].

Lemma 4.2. Assume that $z_{0} \in X_{\frac{1}{2}-\alpha}$. Then, there exists $C>0$ such that

$$
\|z(\tau)\|_{\frac{1}{2}-\alpha}^{2}+\int_{0}^{\tau}\left(\|\dot{z}(s)\|_{-\alpha}^{2}+\|z(s)\|_{1-\alpha}^{2}\right) \mathrm{d} s \leqslant C\left(\int_{0}^{\tau}\|B u(s)\|_{-\alpha}^{2} \mathrm{~d} s+\left\|z_{0}\right\|_{\frac{1}{2}-\alpha}^{2}\right) \quad(\tau>0),
$$

where $z$ is the solution of (1.1)-(1.2).

Proof. Using the facts that

$$
\left\langle\dot{z}(t), A_{0}^{1-2 \alpha} z(t)\right\rangle_{-\alpha, \alpha}=\frac{1}{2} \frac{\mathrm{d}}{\mathrm{d} t}\left\|A_{0}^{\frac{1}{2}-\alpha} z(t)\right\|^{2}, \quad\left\langle A_{0} z(t), A_{0}^{1-2 \alpha} z(t)\right\rangle_{-\alpha, \alpha}=\left\|A_{0}^{1-\alpha} z(t)\right\|^{2},
$$


it follows that if $z$ satisfies the equation (1.1)-(1.2), then

$$
\frac{1}{2} \frac{\mathrm{d}}{\mathrm{d} t}\left\|A_{0}^{\frac{1}{2}-\alpha} z(t)\right\|^{2}+\left\|A_{0}^{1-\alpha} z(t)\right\|^{2}=\left\langle B u(t), A_{0}^{1-2 \alpha} z(t)\right\rangle_{-\alpha, \alpha} .
$$

Integrating the above estimate from 0 to $\tau$ and using the fact that

$$
\left|\left\langle B u(t), A_{0}^{1-2 \alpha} z(t)\right\rangle_{-\alpha, \alpha}\right| \leqslant\|B u(t)\|_{-\alpha}\left\|A_{0}^{1-\alpha} z(t)\right\|,
$$

it follows that

$$
\left\|A_{0}^{\frac{1}{2}-\alpha} z(\tau)\right\|^{2}+\int_{0}^{\tau}\left\|A_{0}^{1-\alpha} z(t)\right\|^{2} \mathrm{~d} t \leqslant\left\|A_{0}^{\frac{1}{2}-\alpha} z_{0}\right\|^{2}+\|B u\|_{L^{2}\left([0, \tau] ; X_{-\alpha}\right)}^{2} .
$$

This leads to:

$$
\|z(\tau)\|_{\frac{1}{2}-\alpha}^{2}+\int_{0}^{\tau}\|z(s)\|_{1-\alpha}^{2} \mathrm{~d} s \leqslant \int_{0}^{\tau}\|B u(s)\|_{-\alpha}^{2} \mathrm{~d} s+\left\|z_{0}\right\|_{\frac{1}{2}-\alpha}^{2} .
$$

Similarly, we have:

$$
\left\langle\dot{z}(t), A_{0}^{-2 \alpha} \dot{z}(t)\right\rangle_{-\alpha, \alpha}=\left\|A_{0}^{-\alpha} \dot{z}(t)\right\|^{2}, \quad\left\langle A_{0} z(t), A_{0}^{-2 \alpha} \dot{z}(t)\right\rangle_{-\alpha, \alpha}=\frac{1}{2} \frac{\mathrm{d}}{\mathrm{d} t}\left\|A_{0}^{\frac{1}{2}-\alpha} z(t)\right\|^{2}
$$

and then

Using again the fact that

$$
\frac{1}{2} \frac{\mathrm{d}}{\mathrm{d} t}\left\|A_{0}^{\frac{1}{2}-\alpha} z(t)\right\|^{2}+\left\|A_{0}^{-\alpha} \dot{z}(t)\right\|^{2}=\left\langle B u(t), A_{0}^{-2 \alpha} \dot{z}(t)\right\rangle_{-\alpha, \alpha} .
$$

$$
\left|\left\langle B u(t), A_{0}^{-2 \alpha} \dot{z}(t)\right\rangle_{-\alpha, \alpha}\right| \leqslant\|B u(t)\|_{-\alpha}\left\|A_{0}^{-\alpha} \dot{z}(t)\right\|,
$$

and integrating by time from 0 to $\tau$, we obtain the following inequality:

$$
\left\|A_{0}^{\frac{1}{2}-\alpha} z(\tau)\right\|^{2}+\int_{0}^{\tau}\left\|A_{0}^{-\alpha} \dot{z}(t)\right\|^{2} \mathrm{~d} t \leqslant\left\|A_{0}^{\frac{1}{2}-\alpha} z_{0}\right\|^{2}+\|B u\|_{L^{2}\left([0, \tau] ; X_{-\alpha}\right)}^{2} .
$$

This leads to:

$$
\|z(\tau)\|_{\frac{1}{2}-\alpha}^{2}+\int_{0}^{\tau}\|\dot{z}(s)\|_{-\alpha}^{2} \mathrm{~d} s \leqslant \int_{0}^{\tau}\|B u(s)\|_{-\alpha}^{2} \mathrm{~d} s+\left\|z_{0}\right\|_{\frac{1}{2}-\alpha}^{2} .
$$

Then (4.1) is deduced from (4.2) and (4.3). This ends the proof.

We give here some convergence results which play an important rule in the proof of our main results.

Lemma 4.3. Let $z_{0} \in X, T>0, \bar{u} \in L^{\infty}([0, T] ; U)$ and $\bar{\tau} \in[0, T]$. Let $\left(u_{n}\right)_{n}$ be a sequence of $L^{\infty}([0, T] ; U)$ and $\left(\tau_{n}\right)_{n}$ be a sequence of $[0, T]$ such that $u_{n} \in L^{\infty}\left([0, T] ; U_{h_{n}}\right)$ for any $n \in \mathbb{N}$ and

$$
\begin{gathered}
u_{n} \rightarrow \bar{u} \quad \text { weakly* in } \quad L^{\infty}([0, T] ; U), \\
\tau_{n} \rightarrow \bar{\tau} \quad \text { in }[0, T] .
\end{gathered}
$$

Then, the following convergence results hold

1. $\lim _{n \rightarrow+\infty}\left\|z\left(., z_{0}, u_{n}\right)-z\left(., z_{0}, \bar{u}\right)\right\|_{C([0, T] ; X)}=0$

2. $\lim _{n \rightarrow+\infty}\left\|z\left(\tau_{n}, z_{0}, u_{n}\right)-z\left(\bar{\tau}, z_{0}, u_{n}\right)\right\|=0$. 
Proof. 1. Denote $\psi(t)=z\left(t, z_{0}, \bar{u}\right)$ and $\psi_{n}(t)=z\left(t, z_{0}, u_{n}\right)$. By the standard energy estimate (4.1), we know that $\left(\psi_{n}\right)_{n}$ is a bounded sequence in

$$
W=C\left([0, T] ; X_{\frac{1}{2}-\alpha}\right) \cap L^{2}\left([0, T] ; X_{1-\alpha}\right) \cap W^{1,2}\left([0, T] ; X_{-\alpha}\right) .
$$

Using Theorem 4.1 with $Y_{0}=X_{\frac{1}{2}-\alpha}, Y_{1}=X$ and $Y_{2}=X_{-\alpha}$, we deduce that:

$$
\exists \widetilde{\psi} \in C([0, T] ; X) \text { s.t. } \psi_{n} \rightarrow \tilde{\psi} \text { strongly in } C([0, T] ; X) \text { and } \psi_{n} \rightarrow \tilde{\psi} \text { weakly in } W \text {. }
$$

Now we prove that $\tilde{\psi}=\psi$. We know that $\psi_{n}$ satisfies:

$$
\begin{aligned}
\dot{\psi}_{n}+A_{0} \psi_{n} & =B u_{n}, \\
\psi_{n}(0) & =z_{0} .
\end{aligned}
$$

Then it is clear that $\dot{\psi}_{n} \rightarrow \dot{\widetilde{\psi}}$ weakly in $L^{2}\left([0, T] ; X_{-\alpha}\right)$ and $A_{0} \psi_{n} \rightarrow A_{0} \tilde{\psi}$ weakly in $L^{2}\left([0, T] ; X_{-\alpha}\right)$ since $\psi_{n} \rightarrow \tilde{\psi}$ weakly in $W$. Moreover, $u_{n} \stackrel{*}{\rightarrow} \bar{u}$ weakly* in $L^{\infty}([0, T] ; U)$ implies that $u_{n} \rightarrow$ $\bar{u}$ weakly in $L^{2}([0, T] ; U)$. Thus, $B u_{n} \rightarrow B \bar{u}$ weakly in $L^{2}([0, T] ; X)$. Finally, $\tilde{\psi}(0)=z_{0}$. Indeed, we know that $z_{0}=\psi_{n}(0) \rightarrow \tilde{\psi}(0)$, since $\psi_{n} \rightarrow \tilde{\psi}$ strongly in $C([0, T] ; X)$.

Consequently, $\tilde{\psi}$ satisfies:

$$
\begin{aligned}
\dot{\tilde{\psi}}+A_{0} \tilde{\psi} & =B \bar{u} \\
\tilde{\psi}(0) & =z_{0},
\end{aligned}
$$

which implies that $\tilde{\psi}=\psi$. This leads to the first assertion.

2. We first notice that

$$
\begin{aligned}
& \left\|z\left(\tau_{n}, z_{0}, u_{n}\right)-z\left(\bar{\tau}, z_{0}, u_{n}\right)\right\| \\
& \leqslant\left\|\mathbb{T}_{\tau_{n}} z_{0}-\mathbb{T}_{\bar{\tau}} z_{0}\right\|+\left\|\int_{0}^{\tau_{n}} \mathbb{T}_{\tau_{n}-\sigma} B u_{n}(\sigma) \mathrm{d} \sigma-\int_{0}^{\bar{\tau}} \mathbb{T}_{\bar{\tau}-\sigma} B u_{n}(\sigma) \mathrm{d} \sigma\right\| \\
& \leqslant\left\|\mathbb{T}_{\tau_{n}} z_{0}-\mathbb{T}_{\bar{\tau}} z_{0}\right\|+\left\|\int_{0}^{\widetilde{\tau_{n}}} \mathbb{T}_{\tau_{n}-\sigma} B u_{n}(\sigma) \mathrm{d} \sigma-\int_{0}^{\tau_{n}} \mathbb{T}_{\bar{\tau}-\sigma} B u_{n}(\sigma) \mathrm{d} \sigma\right\| \\
& \quad+\left\|\int_{\widetilde{\tau_{n}}}^{\tau_{n}} \mathbb{T}_{\tau_{n}-\sigma} B u_{n}(\sigma) \mathrm{d} \sigma\right\|+\left\|\int_{\widetilde{\tau_{n}}}^{\bar{\tau}} \mathbb{T}_{\bar{\tau}-\sigma} B u_{n}(\sigma) \mathrm{d} \sigma\right\| \\
& =L_{1}+L_{2}+L_{3}+L_{4},
\end{aligned}
$$

where $\widetilde{\tau_{n}}=\min \left\{\tau_{n}, \bar{\tau}\right\}$.

By using the continuity of the map $t \mapsto \mathbb{T}_{t} z_{0}$, it is clear that $L_{1}$ converges to zero when $n$ tends to $+\infty$.

Moreover, since $\left(u_{n}\right)_{n} \subset L^{\infty}([0, T], U)$ and using (3.6), we have

$$
\begin{aligned}
L_{3} & \leqslant \int_{\widetilde{\tau_{n}}}^{\tau_{n}}\left\|\mathbb{T}_{\tau_{n}-\sigma} B u_{n}(\sigma)\right\| \mathrm{d} \sigma \\
& \leqslant \int_{\widetilde{\tau_{n}}}^{\tau_{n}}\left\|\mathbb{T}_{\tau_{n}-\sigma} A_{0}^{\alpha}\right\|_{\mathcal{L}(X)}\left\|A_{0}^{-\alpha} B\right\|_{\mathcal{L}(U, X)}\left\|u_{n}(\sigma)\right\|_{U} \mathrm{~d} \sigma \\
& \leqslant C \int_{\widetilde{\tau_{n}}}^{\tau_{n}}\left(\tau_{n}-\sigma\right)^{-\alpha} \mathrm{d} \sigma=\frac{C}{1-\alpha}\left(\tau_{n}-\widetilde{\tau_{n}}\right)^{1-\alpha} \rightarrow 0,
\end{aligned}
$$


since $1-\alpha>0$. Similarly, $L_{4}$ converges to zero.

Now we prove that $L_{2}$ converges to zero. We have

$$
\begin{aligned}
L_{2} & =\left\|\int_{0}^{\widetilde{\tau_{n}}}\left(\mathbb{T}_{\tau_{n}-\sigma}-\mathbb{T}_{\bar{\tau}-\sigma}\right) B\left(u_{n}(\sigma)-\bar{u}(\sigma)\right) \mathrm{d} \sigma\right\|+\left\|\int_{0}^{\widetilde{\tau_{n}}}\left(\mathbb{T}_{\tau_{n}-\sigma}-\mathbb{T}_{\bar{\tau}-\sigma}\right) B \bar{u}(\sigma) \mathrm{d} \sigma\right\| \\
& =L_{2,1}+L_{2,2} .
\end{aligned}
$$

By using the continuity of the map $t \mapsto \mathbb{T}_{t} z_{0}$, it is clear that $L_{2,2}$ converges to zero. Now we prove that $L_{2,1}$ tends to 0 when $n$ tends to $+\infty$.

We have

$$
\begin{aligned}
L_{2,1} & \leqslant\left\|\int_{0}^{\widetilde{\tau_{n}}} \mathbb{T}_{\tau_{n}-\sigma} B\left(u_{n}(\sigma)-\bar{u}(\sigma)\right) \mathrm{d} \sigma\right\|+\left\|\int_{0}^{\widetilde{\tau_{n}}} \mathbb{T}_{\bar{\tau}-\sigma} B\left(u_{n}(\sigma)-\bar{u}(\sigma)\right) \mathrm{d} \sigma\right\| \\
& =\left\|\mathbb{T}_{\tau_{n}-\widetilde{\tau_{n}}}\right\|\left\|z\left(\widetilde{\tau_{n}}, z_{0}, u_{n}\right)-z\left(\widetilde{\tau_{n}}, z_{0}, \bar{u}\right)\right\|+\left\|\mathbb{T}_{\bar{\tau}-\widetilde{\tau_{n}}}\right\|\left\|z\left(\widetilde{\tau_{n}}, z_{0}, u_{n}\right)-z\left(\widetilde{\tau_{n}}, z_{0}, \bar{u}\right)\right\| .
\end{aligned}
$$

Since $\left\|\mathbb{T}_{\tau_{n}-\widetilde{\tau_{n}}}\right\|$ and $\left\|\mathbb{T}_{\bar{\tau}-\widetilde{\tau_{n}}}\right\|$ are bounded, by using the first assertion of this lemma, we can deduce that $L_{2,1}$ converge to zero when $n$ tends to $+\infty$. This ends the proof of the second assertion.

We then give some properties which will be used to prove the convergence results:

\section{Lemma 4.4.}

Denote $\lambda_{1}$ (resp. $\lambda_{1, h}$ ) the first eigenvalue of the operator $A_{0}$ (resp. $A_{0, h}$ ). For every $z_{0} \in X$ and $z_{0, h} \in V_{h}$ the following properties hold

1. $\tau_{0}^{*}\left(z_{0}\right) \leqslant \frac{\ln \left(\left\|z_{0}\right\| / \varepsilon\right)}{\lambda_{1}}$.

2. $\tau_{h}^{*}\left(z_{0, h}\right) \leqslant \frac{\ln \left(\left\|z_{0, h}\right\| / \varepsilon\right)}{\lambda_{1, h}}$.

3. $\lambda_{1} \leqslant \lambda_{1, h}$.

Proof. To prove assertion 1 . we notice that $\left\|z\left(t, z_{0}, 0\right)\right\| \leqslant e^{-\lambda_{1} t}\left\|z_{0}\right\|$ and taking $t=\frac{\ln \left(\left\|z_{0}\right\| / \varepsilon\right)}{\lambda_{1}}$ we obtain that $\left\|z\left(t, z_{0}, 0\right)\right\| \leqslant \varepsilon$. This proves 1 . By a similar argument, it is clear that assertion 2 . holds.

We end by proving the last assertion. In fact, this inequality is easily deduced by the min-max formula: $\lambda_{1}=\min _{z \in X_{\frac{1}{2}}} \frac{\left\|A_{0}^{\frac{1}{2}} z\right\|^{2}}{\|z\|^{2}}$ and $\lambda_{1, h}=\min _{z \in V_{h}} \frac{\left\|A_{0}^{\frac{1}{2}} z\right\|^{2}}{\|z\|^{2}}$. This ends the proof.

Given $\varepsilon>0$ and $z_{0} \in X$ such that $\left\|z_{0}\right\|>\varepsilon$, the lemma below shows that $\tau_{h}^{*}$ has a strictly positive lower and upper bounds, which are independent of $h$.

Lemma 4.5. Assume that $(C 1)$ holds. For every $z_{0} \in X$ such that $\left\|z_{0}\right\|>\varepsilon$, there exist $c, C>0, \tilde{h}>0$ such that for any $h \in(0, \tilde{h})$, we have $c \leqslant \tau_{h}^{*}\left(P_{h} z_{0}\right) \leqslant C$, where $C=2 \frac{\ln \left(\left\|z_{0}\right\| / \varepsilon\right)}{\lambda_{1}}$.

Proof. We begin by proving that $\tau_{h}^{*}\left(P_{h} z_{0}\right)$ is bounded from below. Assume by contradiction that $\lim _{h \rightarrow 0} \tau_{h}^{*}\left(P_{h} z_{0}\right)=0$.

We first notice that

$$
\begin{aligned}
\left\|z_{h}\left(\tau_{h}^{*}, P_{h} z_{0}, u_{h}^{*}\right)-z_{h}\left(0, P_{h} z_{0}, u_{h}^{*}\right)\right\| & =\left\|\mathbb{T}_{\tau_{h}^{*}, h} P_{h} z_{0}+\int_{0}^{\tau_{h}^{*}} \mathbb{T}_{\tau_{h}^{*}-\sigma, h} B u_{h}^{*}(\sigma) \mathrm{d} \sigma-P_{h} z_{0}\right\| \\
& \leqslant\left\|\left(\mathbb{T}_{\tau_{h}^{*}, h}-I d\right) P_{h} z_{0}\right\|+\left\|\int_{0}^{\tau_{h}^{*}} \mathbb{T}_{\tau_{h}^{*}-\sigma, h} B u_{h}^{*}(\sigma) \mathrm{d} \sigma\right\| .
\end{aligned}
$$


We can apply the Trotter-Kato theorem (see, for instance, [2, Theorem 3.6.1, Proposition 3.6.2]), to get that the first term of the right hand side of the previous inequality tends to zero when $h$ tends to zero. Moreover, concerning the left hand side, using (3.7), we have

$$
\begin{aligned}
\left\|\int_{0}^{\tau_{h}^{*}} \mathbb{T}_{\tau_{h}^{*}-\sigma, h} B u_{h}^{*}(\sigma) \mathrm{d} \sigma\right\| & \leqslant \int_{0}^{\tau_{h}^{*}}\left\|\mathbb{T}_{\tau_{h}^{*}-\sigma, h} A_{h}^{\alpha}\right\|_{\mathcal{L}(X)}\left\|A_{h}^{-\alpha} B\right\|_{\mathcal{L}(U, X)}\left\|u_{h}(\sigma)\right\|_{U} \mathrm{~d} \sigma \\
& \leqslant C \int_{0}^{\tau_{h}^{*}}\left(\tau_{h}^{*}-\sigma\right)^{-\alpha} \mathrm{d} \sigma=\frac{C}{1-\alpha}\left(\tau_{h}^{*}\right)^{1-\alpha} \rightarrow 0,
\end{aligned}
$$

since $1-\alpha>0$.

Consequently, we have

$$
\lim _{h \rightarrow 0}\left\|z_{h}\left(\tau_{h}^{*}, P_{h} z_{0}, u_{h}^{*}\right)-z_{h}\left(0, P_{h} z_{0}, u_{h}^{*}\right)\right\|=\lim _{h \rightarrow 0}\left\|z_{h}\left(\tau_{h}^{*}, P_{h} z_{0}, u_{h}^{*}\right)-P_{h} z_{0}\right\|=0 .
$$

Using the fact that $\left\|z_{h}\left(\tau_{h}^{*}, P_{h} z_{0}, u_{h}^{*}\right)\right\| \leqslant \varepsilon$, it is clear that $\lim _{h \rightarrow 0}\left\|P_{h} z_{0}\right\| \leqslant \varepsilon$. However, note that $\lim _{h \rightarrow 0}\left\|P_{h} z_{0}-z_{0}\right\|=0$, which comes from $(C 1)$ since $X_{\gamma}$ is dense in $X$ for any $\gamma>0$ and $P_{h}$ is a projection. This leads to the contradiction with the fact that $\left\|z_{0}\right\|>\varepsilon$.

We prove now that $\tau_{h}^{*}\left(P_{h} z_{0}\right)$ is bounded from above. This is obvious by using Lemma 4.4 , since

$$
\tau_{h}^{*}\left(P_{h} z_{0}\right) \leqslant \frac{\ln \left(\left\|P_{h} z_{0}\right\| / \varepsilon\right)}{\lambda_{1, h}} \leqslant 2 \frac{\ln \left(\left\|z_{0}\right\| / \varepsilon\right)}{\lambda_{1}}<+\infty .
$$

Proof of Theorem 1.1. It suffices to prove the following two inequalities:

$$
\begin{aligned}
& \liminf _{h \rightarrow 0} \tau_{h}^{*}\left(P_{h} z_{0}\right) \geqslant \tau_{0}^{*}\left(z_{0}\right), \\
& \limsup _{h \rightarrow 0} \tau_{h}^{*}\left(P_{h} z_{0}\right) \leqslant \tau_{0}^{*}\left(z_{0}\right) .
\end{aligned}
$$

We begin by proving (4.5). In the following, we denote simply $\tau_{0}^{*}\left(z_{0}\right)$ by $\tau_{0}^{*}$ and $\tau_{h}^{*}\left(P_{h} z_{0}\right)$ by $\tau_{h}^{*}$ when there is no ambiguity. We notice at first the following property:

$$
\forall T>0, \forall u \in \mathcal{U}_{a d}, \tau_{0}^{*}\left(z_{0}\right) \leqslant T+\tau_{0}^{*}\left(z\left(T, z_{0}, u\right)\right) .
$$

We deduce from Theorem 3.6 that

$$
\left\|z\left(\tau_{h}^{*}, z_{0}, u_{h}^{*}\right)-z_{h}\left(\tau_{h}^{*}, P_{h} z_{0}, u_{h}^{*}\right)\right\| \leqslant C h^{\theta} \tau_{h}^{*-1}\left\|z_{0}\right\|_{X}+C h^{\theta(1-\alpha)}\left(|\ln h|+1+\tau_{h}^{*}\right)\left\|u_{h}^{*}\right\|_{L^{\infty}\left([0,+\infty) ; U_{h}\right)},
$$

which leads to:

$$
\begin{aligned}
\left\|z\left(\tau_{h}^{*}, z_{0}, u_{h}^{*}\right)\right\| & \leqslant \varepsilon+C h^{\theta} \tau_{h}^{*-1}\left\|z_{0}\right\|_{X}+C h^{\theta(1-\alpha)}\left(|\ln h|+1+\tau_{h}^{*}\right)\left\|u_{h}^{*}\right\|_{L^{\infty}\left([0,+\infty) ; U_{h}\right)} \\
& \leqslant \varepsilon+C h^{\theta} \tau_{h}^{*-1}+C h^{\theta(1-\alpha)}\left(|\ln h|+1+\tau_{h}^{*}\right) .
\end{aligned}
$$

We denote $\tilde{z_{0}}=z\left(\tau_{h}^{*}, z_{0}, u_{h}^{*}\right)$. According to $(4.7)$ with $T=\tau_{h}^{*}\left(P_{h} z_{0}\right)$, we have:

$$
\tau_{0}^{*}\left(z_{0}\right) \leqslant \tau_{h}^{*}\left(P_{h} z_{0}\right)+\tau_{0}^{*}\left(\widetilde{z_{0}}\right) .
$$


Since $u_{h}^{*} \in L^{\infty}\left([0,+\infty) ; U_{h}\right) \subset L^{\infty}([0,+\infty) ; U)$ and $\left\|u_{h}^{*}(t)\right\| \leqslant 1, u_{h}^{*}$ is then an admissible control for the continuous system. Then, according to Lemma 4.4, we have:

$$
\begin{aligned}
\tau_{0}^{*} & \leqslant \tau_{h}^{*}+\frac{\ln \left(\left(\varepsilon+C h^{\theta} \tau_{h}^{*-1}+C h^{\theta(1-\alpha)}\left(|\ln h|+1+\tau_{h}^{*}\right)\right) / \varepsilon\right)}{\lambda_{1}} \\
& \leqslant \tau_{h}^{*}+\frac{C h^{\theta}}{\lambda_{1} \varepsilon} \tau_{h}^{*-1}+C \frac{h^{\theta(1-\alpha)}}{\lambda_{1} \varepsilon}\left(1+\tau_{h}^{*}\right)+C \frac{h^{\theta(1-\alpha)}|\ln h|}{\lambda_{1} \varepsilon} .
\end{aligned}
$$

Thus, (4.5) can be deduced by taking $h$ to zero and by the fact that $C>\lim _{h \rightarrow 0} \tau_{h}^{*}\left(P_{h} z_{0}\right)>c>0$ (see Lemma 4.5).

We now prove the second inequality (4.6). We have:

$$
\begin{aligned}
& \left\|z_{h}\left(\tau_{0}^{*}, P_{h} z_{0}, Q_{h} u^{*}\right)-z\left(\tau_{0}^{*}, z_{0}, u^{*}\right)\right\| \\
& \leqslant\left\|z_{h}\left(\tau_{0}^{*}, P_{h} z_{0}, Q_{h} u^{*}\right)-z_{h}\left(\tau_{0}^{*}, P_{h} z_{0}, u^{*}\right)\right\|+\left\|z_{h}\left(\tau_{0}^{*}, P_{h} z_{0}, u^{*}\right)-z\left(\tau_{0}^{*}, z_{0}, u^{*}\right)\right\| \\
& \leqslant\left\|z_{h}\left(\tau_{0}^{*}, P_{h} z_{0}, Q_{h} u^{*}\right)-z_{h}\left(\tau_{0}^{*}, P_{h} z_{0}, u^{*}\right)\right\|+C h^{\theta} \tau_{0}^{*-1}+C h^{\theta(1-\alpha)}\left(|\ln h|+1+\tau_{0}^{*}\right),
\end{aligned}
$$

using (3.9). Denote $f(h)=\left\|z_{h}\left(\tau_{0}^{*}, P_{h} z_{0}, Q_{h} u^{*}\right)-z_{h}\left(\tau_{0}^{*}, P_{h} z_{0}, u^{*}\right)\right\|$. Notice that $\lim _{h \rightarrow 0} f(h)=0$. Indeed,

$$
\lim _{h \rightarrow 0} f(h)=\lim _{h \rightarrow 0}\left\|z_{h}\left(\tau_{0}^{*}, P_{h} z_{0}, Q_{h} u^{*}\right)-z_{h}\left(\tau_{0}^{*}, P_{h} z_{0}, u^{*}\right)\right\|=\lim _{h \rightarrow 0}\left\|\Phi_{\tau^{*}, h}\left(u^{*}-Q_{h} u^{*}\right)\right\| .
$$

Moreover, we have

$$
\left\|\Phi_{\tau^{*}, h}\left(u^{*}-Q_{h} u^{*}\right)\right\| \leqslant\left\|\left(\Phi_{\tau^{*}, h}-\Phi_{\tau^{*}}\right)\left(u^{*}-Q_{h} u^{*}\right)\right\|+\left\|\Phi_{\tau^{*}}\left(u^{*}-Q_{h} u^{*}\right)\right\| .
$$

Since $B \in \mathcal{L}\left(U, X_{-\alpha}\right)$, with $0 \leqslant \alpha<\frac{1}{2}$, it is known that $\Phi_{\tau^{*}} \in \mathcal{L}\left(L^{2}\left(\left[0, \tau^{*}\right] ; U\right), X\right)$ (see for instance [31, Proposition 5.1.3]). Combining this fact with assertion 7. in Proposition 3.5 and with assumption $(C 5)$, it is clear that

$$
\lim _{h \rightarrow 0} f(h)=0 .
$$

Thus, we have:

$$
\left\|z_{h}\left(\tau_{0}^{*}, P_{h} z_{0}, Q_{h} u^{*}\right)\right\| \leqslant \varepsilon+f(h)+C h^{\theta} \tau_{0}^{*-1}+C h^{\theta(1-\alpha)}\left(|\ln h|+1+\tau_{0}^{*}\right) .
$$

By the similar argument as in (4.8), we have:

$$
\begin{aligned}
\tau_{h}^{*}\left(P_{h} z_{0}\right) & \leqslant \tau_{0}^{*}+\frac{\ln \left(\left(\varepsilon+f(h)+C h^{\theta} \tau_{0}^{*-1}+C h^{\theta(1-\alpha)}\left(|\ln h|+1+\tau_{0}^{*}\right)\right) / \varepsilon\right)}{\lambda_{1, h}} \\
& \leqslant \tau_{0}^{*}+\frac{f(h)+C h^{\theta} \tau_{0}^{*-1}+C\left(1+\tau_{0}^{*}\right) h^{\theta(1-\alpha)}+C h^{\theta(1-\alpha)}|\ln h|}{\lambda_{1} \varepsilon}
\end{aligned}
$$

This leads to inequality (4.6) by taking $h$ to zero.

Proof of Theorem 1.2. Denote $T=2 \frac{\ln \left(\left\|z_{0}\right\| / \varepsilon\right)}{\lambda_{1}}$. It is clear that $\tau_{h}^{*} \leqslant T$ for all $h>0$ and $\tau_{0}^{*} \leqslant T$ (see Lemma 4.4 and Lemma 4.5). We extend $\left(u_{h}^{*}\right)_{h}$ and $u_{0}^{*}$ to time $T$ by zero.

Since $\left\|u_{h}^{*}\right\|_{L^{\infty}\left([0, T] ; U_{h}\right)} \leqslant 1$, there exist a control $\bar{u} \in L^{\infty}([0, T] ; U)$ and a subsequence $\left(h_{n}\right)_{n} \rightarrow 0$, such that:

$$
u_{h_{n}}^{*} \rightarrow \bar{u} \quad \text { weakly* in } \quad L^{\infty}([0, T] ; U) .
$$

Now we prove that $\bar{u}=u_{0}^{*}$. 
The main step here is to prove the following convergence property:

$$
\left\|z_{h_{n}}\left(\tau_{h_{n}}^{*}, P_{h_{n}} z_{0}, u_{h_{n}}^{*}\right)-z\left(\tau_{0}^{*}, z_{0}, \bar{u}\right)\right\| \rightarrow 0 .
$$

Indeed, since $\bar{B}(0, \varepsilon)$ is complete (notice that $\overline{B_{h}}(0, \varepsilon) \subset \bar{B}(0, \varepsilon)$ ), (4.9) leads to $z\left(\tau_{0}^{*}, z_{0}, \bar{u}\right) \in \bar{B}(0, \varepsilon)$. Then, by the uniqueness of the time optimal control and Theorem 1.1, we deduce that $\bar{u}=u_{0}^{*}$.

We are then reduced to prove (4.9). We have:

$$
\begin{aligned}
\left\|z_{h_{n}}\left(\tau_{h_{n}}^{*}, P_{h_{n}} z_{0}, u_{h_{n}}^{*}\right)-z\left(\tau_{0}^{*}, z_{0}, \bar{u}\right)\right\| & \leqslant\left\|z_{h_{n}}\left(\tau_{h_{n}}^{*}, P_{h_{n}} z_{0}, u_{h_{n}}^{*}\right)-z\left(\tau_{h_{n}}^{*}, z_{0}, u_{h_{n}}^{*}\right)\right\| \\
& +\left\|z\left(\tau_{h_{n}}^{*}, z_{0}, u_{h_{n}}^{*}\right)-z\left(\tau_{0}^{*}, z_{0}, u_{h_{n}}^{*}\right)\right\| \\
& +\left\|z\left(\tau_{0}^{*}, z_{0}, u_{h_{n}}^{*}\right)-z\left(\tau_{0}^{*}, z_{0}, \bar{u}\right)\right\| .
\end{aligned}
$$

The first term of the right hand side of (4.10) converges to zero using the error estimate (3.9). Moreover, by using the first and the second assertions in Lemma 4.3, it is clear that the second and the third terms of the right hand of (4.10) converges to zero. We then deduce (4.9).

Thus, we have:

$$
u_{h_{n}}^{*} \rightarrow u_{0}^{*} \text { weakly* in } L^{\infty}([0, T] ; U) .
$$

We deduce immediately that:

$$
u_{h_{n}}^{*} \rightarrow u_{0}^{*} \text { weakly in } L^{2}([0, T] ; U) \text {. }
$$

At last, since both $u_{h_{n}}^{*}$ and $u_{0}^{*}$ are bang-bang controls (see Corollary 2.4), we have

$$
\lim _{n \rightarrow+\infty}\left\|u_{h_{n}}^{*}\right\|_{L^{2}([0, T] ; U)}=\left\|u_{0}^{*}\right\|_{L^{2}([0, T] ; U)} .
$$

According to the Radon-Riesz property, this leads to the strong convergence in $L^{2}([0, T] ; U)$. This ends the proof.

\section{Applications}

Example 5.1. Let $\Omega \subset \mathbb{R}^{N}$ be a bounded open set with a boundary $\partial \Omega$ of class $C^{2}$. For each $z_{0} \in L^{2}(\Omega)$ and $u \in L^{\infty}\left([0,+\infty) ; L^{2}(\Omega)\right)$, we consider the following initial and boundary value problem

$$
\begin{aligned}
\dot{z}(x, t)-\triangle z(x, t) & =\chi_{\omega}(x) u(x, t) & & (x \in \Omega, t \geqslant 0), \\
z(x, t) & =0 & & (x \in \partial \Omega, t \geqslant 0), \\
z(x, 0) & =z_{0}(x) & & (x \in \Omega),
\end{aligned}
$$

where $\omega \subset \Omega$ is an open and non-empty subset with its characteristic function $\chi_{\omega}$. The system (5.1)(5.3) can be formulated in the abstract form (1.1)-(1.2) by taking $A_{0}=-\triangle$ with Dirichlet boundary conditions, of domain $D\left(A_{0}\right)=H_{0}^{1}(\Omega) \cap H^{2}(\Omega)$ on $X=L^{2}(\Omega)$. The control operator $B \in \mathcal{L}(U, X)$ (here $\alpha=0$ ) is defined by

$$
B \varphi=\chi_{\omega} \tilde{\varphi},
$$

where $U=L^{2}(\omega)$ and where $\tilde{\varphi}$ is the extension by 0 of $\varphi$ on $\Omega$ outside $\omega$. 
We then consider the standard $P_{1}$ finite element method with regular triangulation $\mathcal{T}_{h}$ of $\Omega$ (see for example, Thomée [28], Raviart and Thomas [25]) and we build the finite element space $V_{h} \subset H_{0}^{1}(\Omega)$ of $X$, defined by

$$
V_{h}=\left\{\varphi \in C(\bar{\Omega}) \mid \varphi_{\mid \mathcal{T}} \in P_{1}(\mathcal{T}) \quad \text { for every } \quad \mathcal{T} \in \mathcal{T}_{h}, \varphi_{\mid \partial \Omega}=0\right\}
$$

where $P_{1}(\mathcal{T})$ is the set of affine functions on $\mathcal{T}$. We also define $U_{h}$ a finite dimensional subspace of $U$ by

$$
U_{h}:=\left\{\chi_{\omega} \varphi_{h} \mid \varphi_{h} \in V_{h}\right\} .
$$

Denote $P_{h}$ the $L^{2}$-projection from $L^{2}(\Omega)$ to $V_{h}$, and we consider the following space semi-discrete scheme formulated in the form of (1.5)-(1.6):

$$
\begin{array}{rlrl}
\dot{z}_{h}(t)+A_{h} z_{h}(t) & =B_{h} u_{h}(t) & & (t \geqslant 0), \\
z_{h}(0) & =P_{h} z_{0} & \left(z_{0} \in X\right),
\end{array}
$$

where $u_{h}$ is taken in $L^{\infty}\left([0,+\infty) ; U_{h}\right)$ and the operator $A_{h}$ and $B_{h}$ are defined by

$$
<A_{h} \varphi, \psi>=\int_{\Omega} \nabla \varphi \nabla \psi \mathrm{d} x \quad\left(\varphi, \psi \in V_{h}\right)
$$

and $B_{h} \in \mathcal{L}\left(U, V_{h}\right)$ is defined by :

$$
\left\langle B_{h} u, \varphi\right\rangle=\left\langle u, B^{*} \varphi\right\rangle_{U} \quad\left(\varphi \in V_{h}, u \in U\right) .
$$

Given $\varepsilon>0$ with the same notation as in Section 1, we consider the time optimal control problem (TP) associated to (5.1)-(5.3) (resp. ( $\left.\mathbf{T P}_{\mathbf{h}}\right)$ associated to (5.4)-(5.5)) and denote $\left(\tau_{0}^{*}, u_{0}^{*}\right)$ the corresponding optimal time and time-optimal control (resp. $\left.\left(\tau_{h}^{*}, u_{h}^{*}\right)\right)$.

We can now state the following convergence results:

Proposition 5.2. With the notation above, let, for every $\varepsilon>0, z_{0} \in X$ such that $\left\|z_{0}\right\|>\varepsilon$. Then we have that $\tau_{h}^{*} \rightarrow \tau_{0}^{*}$, and $u_{h}^{*} \rightarrow u_{0}^{*}$ in $L^{2}\left([0, T] ; L^{2}(\Omega)\right)$.

Proof. In order to apply Theorem 1.1 and Theorem 1.2, we verify that conditions $(C 1)-(C 5)$ are satisfied with $\alpha=0$ and $\theta=2$. Indeed, $(C 1)$ is a standard error estimate when applying the $P_{1}$ finite element scheme (see for example [28]). $(C 2)$ is proved in Bramble et al [6] and in Lasiecka [18]. Moreover, $(C 3)-(C 5)$ clearly hold true because here the control operator $B$ is bounded. Furthermore, it is known that the assumption made in Theorem 1.2 holds true (see for example [23]). Thus, we can apply Theorem 1.1 and Theorem 1.2 to conclude.

Example 5.3. Let $\Omega \subset \mathbb{R}^{N}$ be a bounded open set with a boundary $\partial \Omega$ of class $C^{2}$. We consider the heat equation

$$
\dot{z}(x, t)-\triangle z(x, t)=0 \quad(x \in \Omega, \quad t \geqslant 0),
$$

with the initial and boundary conditions

$$
\begin{aligned}
\left.\frac{\partial z}{\partial \nu}\right|_{\partial \Omega}(x, t) & =u(x, t) \quad(x \in \partial \Omega, \quad t \geqslant 0), \\
z(x, 0) & =z_{0}(x) \in L^{2}(\Omega) \quad(x \in \Omega) .
\end{aligned}
$$


It is known that the system (5.8)-(5.10) can be written in the form of (1.1)-(1.2) by taking $X=$ $L^{2}(\Omega), U=L^{2}(\partial \Omega), D\left(A_{0}\right)=\left\{z \in H^{2}(\Omega)\left|\frac{\partial z}{\partial \nu}\right|_{\partial \Omega}=0\right\}, A_{0}=-\triangle$ and the control operator $B \in$ $\mathcal{L}\left(U, X_{-\alpha}\right)$ (here $\left.\alpha=\frac{1}{4}+\varepsilon, \varepsilon>0\right)$ defined by

$$
B u=A_{0} N u \quad(u \in U),
$$

where $N$ is the Neumann map. This map is defined by $N v=z$, where $z \in L^{2}(\Omega)$ is the unique solution of the nonhomogeneous elliptic equation

$$
\begin{cases}\triangle z=0 & \text { in } \Omega \\ \left.\frac{\partial z}{\partial \nu}\right|_{\partial \Omega}=v & \text { on } \partial \Omega\end{cases}
$$

We refer to [19, Par. 5] which shows that the Galerkin semi-discrete approximation of system (5.8)-(5.10) satisfies conditions $(C 1)-(C 5)$ (and for other numerical approximation method).

We can obtain the following result:

Proposition 5.4. With the notation above, let, for every $\varepsilon>0, z_{0} \in X$ such that $\left\|z_{0}\right\|>\varepsilon$. Then we have that $\tau_{h}^{*} \rightarrow \tau_{0}^{*}$, and $u_{h}^{*} \rightarrow u_{0}^{*}$ in $L^{2}\left([0, T] ; L^{2}(\partial \Omega)\right)$.

Proof. In order to apply Theorem 1.1 and Theorem 1.2, we verify that conditions $(C 1)-(C 5)$ are satisfied with $\alpha=\frac{1}{4}+\varepsilon, \varepsilon>0$ and $\theta=2$ (see for example [19, Ch. 5]). Moreover, it is known that the assumption made in Theorem 1.2 holds true (see for example [20, Section 3.3, Ch. 5.3]). Thus, we can apply Theorem 1.1 and Theorem 1.2 to conclude.

We illustrate in the follows some numerical results for the 1d-heat equation with Neumann boundary condition. More precisely, we take $\Omega=(0,1), \epsilon=1$ (the radius of the target ball), control constraints $|u(t, 0)| \leqslant 1$ and $|u(t, 1)| \leqslant 1$ and the initial data $z_{0}(x)=5 \sin (\pi x)$. We choose to discretize totally the system, with the implicit Euler method for time and finite difference scheme for space. We also use the optimization solver IPOPT in Matlab.

We obtain the following result:

\begin{tabular}{|c|cccccc|}
\hline Number of discretisation & 10 & 20 & 40 & 60 & 80 & 100 \\
\hline time optimal & 1.3442 & 1.3469 & 1.3464 & 1.3459 & 1.3456 & 1.3454 \\
\hline
\end{tabular}

Moreover, we find that $u_{h}(t, 0)=u_{h}(t, 1)=-1$ for any $t>0$ and for any number of discretization, that validates the fact that the time optimal control for the discrete system converges towards a bang-bang time optimal control.

Remark 5.5. The error estimates of the time and control convergence remain to study. 


\section{References}

[1] N. Arada and J.-P. Raymond. Time optimal problems with Dirichlet boundary controls. Discrete Contin. Dyn. Syst., 9(6):1549-1570, 2003.

[2] Wolfgang Arendt, Charles J. K. Batty, Matthias Hieber, and Frank Neubrander. Vector-valued Laplace transforms and Cauchy problems, volume 96 of Monographs in Mathematics. Birkhäuser Verlag, Basel, 2001.

[3] M. Badra. Stabilisation par feedback et approximation des équations de Navier-Stokes. PhD thesis, 2006. Thèse de doctorat dirigée par Jean-Pierre Raymond, Mathématiques appliquées, Toulouse $3,2006$.

[4] V. Barbu. Analysis and control of nonlinear infinite-dimensional systems, volume 190 of Mathematics in Science and Engineering. Academic Press, Inc., Boston, MA, 1993.

[5] V. Barbu. Nonlinear differential equations of monotone types in Banach spaces. Springer Monographs in Mathematics. Springer, New York, 2010.

[6] J. H. Bramble, A. H. Schatz, V. Thomée, and L. B. Wahlbin. Some convergence estimates for semidiscrete Galerkin type approximations for parabolic equations. SIAM J. Numer. Anal., 14(2):218-241, 1977.

[7] H. Brezis. Analyse fonctionnelle. Collection Mathématiques Appliquées pour la Maîtrise. [Collection of Applied Mathematics for the Master's Degree]. Masson, Paris, 1983. Théorie et applications. [Theory and applications].

[8] O. Cârjă. On variational perturbations of control problems: minimum-time problem and minimum-effort problem. J. Optim. Theory Appl., 44(3):407-433, 1984.

[9] O. Cârjă. The time optimal problem for boundary-distributed control systems. Boll. Un. Mat. Ital. $B(6), 3(3): 563-581,1984$.

[10] C. Castro and E. Zuazua. Some topics on the control and homogenization of parabolic partial differential equations. In Homogenization, 2001 (Naples), volume 18 of GAKUTO Internat. Ser. Math. Sci. Appl., pages 49-93. Gakkōtosho, Tokyo, 2003.

[11] H. O. Fattorini. Time-optimal control of solutions of operational differential equations. Journal of the Society for Industrial and Applied Mathematics Series A Control, 2(1):54-59, 1964.

[12] H. O. Fattorini. Infinite Dimensional Linear Control Systems. The Time Optimal and Norm Optimal Control Problems. North-Holland Mathematics Studies, 201. Elsevier, Amsterdam, 2005.

[13] H.O. Fattorini. Infinite Dimensional Optimization and Control Theory. Cambridge Studies in Advanced Mathematics. Cambridge University Press, 1999.

[14] G. P. Henze, C. Felsmann, and G. Knabe. Evaluation of optimal control for active and passive building thermal storage. International Journal of Thermal Sciences, 43(2):173-183, 2004.

[15] G. Knowles. Finite element approximation of parabolic time optimal control problems. SIAM J. Control Optim., 20(3):414-427, 1982. 
[16] K. Kunisch and D. Wachsmuth. On time optimal control of the wave equation, its regularization and optimality system. ESAIM Control Optim. Calc. Var., 19(2):317-336, 2013.

[17] K. Kunisch and L. Wang. Time optimal control of the heat equation with pointwise control constraints. ESAIM Control Optim. Calc. Var., 19(2):460-485, 2013.

[18] I. Lasiecka. Convergence estimates for semidiscrete approximations of nonselfadjoint parabolic equations. SIAM J. Numer. Anal., 21(5):894-909, 1984.

[19] I. Lasiecka. Galerkin approximations of abstract parabolic boundary value problems with rough boundary data- $l_{p}$ theory. Mathematics of Computation, 47(175):pp. 55-75, 1986.

[20] I. Lasiecka and R. Triggiani. Control Theory for Partial Differential Equations: Continuous and Approximation Theories, Volume 1, Abstract Parabolic Systems, volume 1. Cambridge University Press, 2000.

[21] X.J. Li and J.M. Yong. Optimal control theory for infinite-dimensional systems. Systems \& Control: Foundations \& Applications. Birkhäuser Boston Inc., Boston, MA, 1995.

[22] J.-L. Lions. Contrôle optimal de systèmes gouvernés par des équations aux dérivées partielles. Avant propos de P. Lelong. Dunod, Paris, 1968.

[23] S. Micu, I. Roventa, and M. Tucsnak. Time optimal boundary controls for the heat equation. J. Funct. Anal., 263(1):25-49, 2012.

[24] A. Pazy. Semigroups of Linear Operators and Applications to Partial Differential Equations. Number vol. 44 in Applied Mathematical Sciences. Springer, 1992.

[25] P.A. Raviart and J.M. Thomas. Introduction à l'analyse numérique des équations aux dérivées partielles. Collection Mathématiques appliquées pour la maîtrise. Dunod, 2004.

[26] J. Simon. Compact sets in the space $L^{p}(0, T ; B)$. Ann. Mat. Pura Appl. (4), 146:65-96, 1987.

[27] L. Tebou. Uniform null controllability of the heat equation with rapidly oscillating periodic density. C. R. Math. Acad. Sci. Paris, 347(13-14):779-784, 2009.

[28] V. Thomée. Galerkin finite element methods for parabolic problems, volume 25 of Springer Series in Computational Mathematics. Springer-Verlag, Berlin, second edition, 2006.

[29] F. Tröltzsch. On generalized bang-bang principles for two time-optimal heating problems with constraints on the control and the state. Demonstratio Math., 15(1):131-143, 1982.

[30] M. Tucsnak, J. Valein, and C.-T. Wu. Numerical approximation of some time optimal control problems. In Control Conference (ECC), 2015 European, pages 1339-1342. IEEE, 2015.

[31] M. Tucsnak and G. Weiss. Observation and control for operator semigroups. Birkhäuser Advanced Texts: Basler Lehrbücher. [Birkhäuser Advanced Texts: Basel Textbooks]. Birkhäuser Verlag, Basel, 2009.

[32] G. Wang and L. Wang. Finite element approximations of optimal controls for the heat equation with end-point state constraints. Int. J. Numer. Anal. Model., 9(4):844-875, 2012.

[33] G. Wang and G. Zheng. An approach to the optimal time for a time optimal control problem of an internally controlled heat equation. SIAM J. Control Optim., 50(2):601-628, 2012. 